\title{
Simple completeness proofs for some spatial logics of the real line
}

\author{
Ian Hodkinson
}

December 26, 2012

\begin{abstract}
McKinsey-Tarski (1944), Shehtman (1999), and Lucero-Bryan (2011) proved completeness theorems for modal logics with modalities $\square, \square$ and $\forall$, and $[\partial]$ and $\forall$, respectively, with topological semantics over the real numbers. We give short proofs of these results using lexicographic sums of linear orders.
\end{abstract}

\section{Introduction}

This paper contains no new results at all. Its sole aim is to present what I believe are new and simple completeness proofs of some modal logics of the real line $\mathbb{R}$. They are often regarded as spatial logics - see [1] for example. The paper is deliberately kept short, with little historical background. There are three main theorems:

1. If $\square$ is read as the interior operator in the standard topology on $\mathbb{R}$, the logic of $\mathbb{R}$ is $\mathrm{S} 4$ - proved by McKinsey-Tarski [13]. This result was the first in the field. Interest in it is undergoing a renaissance and several alternative proofs have recently appeared $[14,15,2,1,10,8]$. So yet another proof will do no harm and may be of interest.

2. The logic of $\mathbb{R}$ with $\square$ and the universal modality $\forall$ is S4UC — proved by Shehtman [19].

3. If we replace $\square$ by a different box $[\partial]$, to be read as the coderivative operator, then the logic of $\mathbb{R}$ with $[\partial]$ and $\forall$ is $\mathrm{KD}_{4} \mathrm{G}_{2} . \mathrm{UC}$ - proved by Lucero-Bryan [12].

The logic of $\mathbb{R}$ with $[\partial]$ alone is $\mathrm{KD} \mathrm{G}_{2}$ : this was proved by Shehtman [21], and later by Lucero-Bryan [12]. We will not prove it here. It can be done by removing parts of the proof of (3), which the reader may wish to do.

One may wonder whether the proofs would go through with $\forall$ replaced by the stronger difference operator $[\neq]$. However, Kudinov [9] has shown that the $\operatorname{logic}$ of $\mathbb{R}$ with $\square$ and $[\neq]$ is not finitely axiomatisable, and his argument appears to work for $[\partial]$ and $[\neq]$ as well.

Completeness proofs for modal logics with topological semantics over $\mathbb{R}$ often start by applying methods from classical modal logic, of varying sophistication, and end by applying topological techniques. Our proof proceeds like this as well, 
but with two differences. First, our use of modal logic is relatively straightforward. All we need is the finite model property for the logics, so that we can argue by induction on the size of parts of the finite model. For some of the logics the finite model property is nontrivial to establish, but we have nothing new to contribute here so we omit proofs and simply cite the literature. (It is worth noting here that we presuppose some familiarity with basic modal logic.) Second, we use very little topology. Instead, we use lexicographic sums of linear orders. Although these are very well known in some circles, a substantial part of the paper is devoted to introducing them, in the hope that they become known a little more widely, and to make the paper more self-contained.

The layout of the paper is simple. We describe syntax and semantics in $\S 2$ and lexicographic sums in $\S 3$. The three completeness proofs are in $\S \S 4-6$, and we conclude in $\S 7$ with a couple of open questions.

We use standard notation such as $\mathbb{Z}, \mathbb{Q}, \mathbb{R}$. We often identify (notationally) a structure with its domain. For a map $f: X \rightarrow Y$ and subsets $X^{\prime} \subseteq X$, $Y^{\prime} \subseteq Y$, we write $f\left(X^{\prime}\right)=\left\{f(x): x \in X^{\prime}\right\}, \operatorname{rng}(f)=f(X)$, and $f^{-1}\left(Y^{\prime}\right)=$ $\left\{x \in X: f(x) \in Y^{\prime}\right\}$. The cardinality of a set $X$ is denoted by $|X|$.

\section{Definitions}

We will study the logic of $\mathbb{R}$ in three sublanguages of the following ambient language $\mathcal{L}$. We fix a countably infinite set $P V$ of propositional variables (or 'atoms').

\subsection{Syntax $-\mathcal{L}$-formulas}

The formulas of $\mathcal{L}$ are as follows:

1. $\top$ is an $\mathcal{L}$-formula.

2. Any atom $p \in P V$ is an $\mathcal{L}$-formula.

3. If $\varphi, \psi$ are $\mathcal{L}$-formulas then so are $\neg \varphi$ and $(\varphi \wedge \psi)$.

4. If $\varphi$ is an $\mathcal{L}$-formula then $\square \varphi,[\partial] \varphi$, and $\forall \varphi$ are also $\mathcal{L}$-formulas.

We will write $\mathcal{L}$ for the set of all $\mathcal{L}$-formulas, $\mathcal{L}_{\square}$ for the set of $\mathcal{L}$-formulas not involving $[\partial]$ or $\forall, \mathcal{L}_{[\partial]}$ for the set of $\mathcal{L}$-formulas not involving $\square$ or $\forall$, $\mathcal{L}_{\square \forall}$ for the set of $\mathcal{L}$-formulas not involving $[\partial]$, and $\mathcal{L}_{[\partial] \forall}$ for the set of $\mathcal{L}$ formulas not involving $\square$. We will use the standard abbreviations: $\perp=\neg \top$, $\varphi \vee \psi=\neg(\neg \varphi \wedge \neg \psi), \varphi \rightarrow \psi=\neg(\varphi \wedge \neg \psi), \diamond \varphi=\neg \square \neg \varphi,\langle\partial\rangle \varphi=\neg[\partial] \neg \varphi$, and $\exists \varphi=\neg \forall \neg \varphi$. We adopt the usual binding conventions for the connectives and omit parentheses where no ambiguity results.

\section{$2.2 \quad$ Kripke semantics}

Although Kripke semantics is not the main concern of the paper, our proofs will use Kripke semantics for $\mathcal{L}$-formulas. A binary relation on a set $W$ is a subset $R \subseteq W \times W$. We will write any of $R(w, u), R w u$, and $w R u$ to denote that $(w, u) \in R$. For $w \in W$, we write $R(w)$ for the set $\{u \in W: R w u\}$. For $X \subseteq W$ we write $R \uparrow X$ for the binary relation $R \cap(X \times X)$ on $X$. 
A Kripke frame is a pair $\mathcal{F}=(W, R)$, where $W$ is a nonempty set and $R$ a binary relation on $W$. A Kripke frame $\left(W^{\prime}, R^{\prime}\right)$ is said to be a generated subframe of $\mathcal{F}$ if $W^{\prime} \subseteq W, R^{\prime}=R \uparrow W^{\prime}$, and $R(w) \subseteq W^{\prime}$ for every $w \in W^{\prime}$. An assignment into $\mathcal{F}$ is a map $g: P V \rightarrow \wp(W)$, where $\wp$ denotes the power set operation, and a Kripke model is a triple $(W, R, g)$, where $\mathcal{F}=(W, R)$ is a Kripke frame and $g$ an assignment into $\mathcal{F}$.

For a Kripke model $\mathcal{M}=(W, R, g)$ an element $w \in W$, and a formula $\varphi \in \mathcal{L}$, we define $\mathcal{M}, w=\varphi$ (' $\varphi$ is true in $\mathcal{M}$ at $w$ ') by induction on $\varphi$ as follows:

1. $\mathcal{M}, w \models \top$

2. $\mathcal{M}, w \models p$ iff $w \in g(p)$, for $p \in P V$

3. $\mathcal{M}, w \models \neg \varphi$ iff $\mathcal{M}, w \not \models \varphi$

4. $\mathcal{M}, w \models \varphi \wedge \psi$ iff $\mathcal{M}, w \models \varphi$ and $\mathcal{M}, w \models \psi$

5. $\mathcal{M}, w \models \square \varphi$ iff $\mathcal{M}, u \models \varphi$ for every $u \in R(w)$

6. $\mathcal{M}, w \models[\partial] \varphi$ iff $\mathcal{M}, u=\varphi$ for every $u \in R(w)$

7. $\mathcal{M}, w \models \forall \varphi$ iff $\mathcal{M}, u \models \varphi$ for every $u \in W$

We make no distinction between $\square$ and $[\partial]$ in Kripke semantics. We will always consider the two boxes separately, so this will not be a problem for us.

As usual, an $\mathcal{L}$-formula $\varphi$ is said to be satisfied in a Kripke model $\mathcal{M}=$ $(W, R, h)$ if $\mathcal{M}, w \models \varphi$ for some $w \in W$, and valid in a Kripke frame $\mathcal{F}=(W, R)$ if $(W, R, h), w=\varphi$ for every assignment $h$ into $\mathcal{F}$ and every $w \in W$.

\subsection{Linear orders}

A linear order is a structure $(I,<)$, where $I$ is a nonempty set and $<$ a binary relation on $I$ with the following properties:

1. $\forall x \neg(x<x)$

irreflexivity

2. $\forall x y z(x<y \wedge y<z \rightarrow x<z)$

transitivity

3. $\forall x y(x<y \vee x=y \vee y<x)$

linearity

We let $x \leq y$ abbreviate $x<y \vee x=y$ as usual. In line with our general convention, we will often identify (notationally) a linear order $(I,<)$ with its domain $I$. For example, $(\mathbb{Z},<)$ and $(\mathbb{R},<)$ are linear orders, and we often write them simply as $\mathbb{Z}, \mathbb{R}$. A subset $D \subseteq I$ is said to be dense if for every $i, j \in I$ with $i<j$, there is $d \in D$ with $i<d<j$. The order $I$ itself is dense if $I$ is a dense subset of $I$. Linear orders $(I,<),\left(I^{\prime},<^{\prime}\right)$ are said to be isomorphic (in symbols, $\left.(I,<) \cong\left(I^{\prime},<^{\prime}\right)\right)$ if there is a bijection $f: I \rightarrow I^{\prime}$ such that $i<j$ iff $f(i)<^{\prime} f(j)$ for all $i, j \in I$; we say that $f: I \rightarrow I^{\prime}$ is an isomorphism. 


\subsection{Linear models}

We give $\mathcal{L}$-formulas semantics over a linear order $(I,<)$ as follows. An assignment (into $I$ ) is a map $h: P V \rightarrow \wp(I)$. A linear model (over $I$ ) is a triple $M=(I,<, h)$, where $(I,<)$ is a linear order and $h$ an assignment into $I$. We write $\operatorname{dom}(M)$ (the domain of $M$ ) for the set $I$, and $\operatorname{supp}(M)$ (the support of $M)$ for the set $\{p \in P V: h(p) \neq \emptyset\}$. For a linear order $\left(I^{\prime},<^{\prime}\right)$ and a linear model $M^{\prime}=\left(I^{\prime},<^{\prime}, h^{\prime}\right)$, we say that $M$ is isomorphic to $M^{\prime}$, and write $M \cong M^{\prime}$, if there is an isomorphism $f:(I,<) \rightarrow\left(I^{\prime},<^{\prime}\right)$ with $h^{\prime}(p)=f(h(p))$ for every $p \in P V$. We say that $M^{\prime}$ is a submodel of $M$, and write $M^{\prime} \subseteq M$, if $I^{\prime} \subseteq I$, $<^{\prime}=<\uparrow I^{\prime}$, and $h^{\prime}(p)=h(p) \cap I^{\prime}$ for every $p \in P V$. We say that $M^{\prime}$ is an initial submodel of $M$ if $M^{\prime} \subseteq M$ and whenever $i \in I, i^{\prime} \in I^{\prime}$, and $i<i^{\prime}$, we have $i \in I^{\prime}$. We say that $M^{\prime}$ is a final submodel of $M$ if $M^{\prime} \subseteq M$ and whenever $i \in I, i^{\prime} \in I^{\prime}$, and $i^{\prime}<i$, we have $i \in I^{\prime}$.

For a linear model $M=(I,<, h)$ and a point $x \in I$, we define $M, x \models \varphi$ by induction on $\varphi$, as follows:

1. $M, x \mid=\top$

2. $M, x \mid=p$ iff $x \in h(p)$, for $p \in P V$

3. $M, x \mid=\neg \varphi$ iff $M, x \not=\varphi$

4. $M, x \mid=\varphi \wedge \psi$ iff $M, x \models \varphi$ and $M, x \models \psi$

5. $M, x \mid=\square \varphi$ iff there exist $y, z \in I$ with $y<x<z$ and such that $M, t=\varphi$ for all $t \in I$ with $y<t<z$

6. $M, x=[\partial] \varphi$ iff there exist $y, z \in I$ with $y<x<z$ and such that $M, t=\varphi$ for all $t \in I$ with $y<t<z$ and $t \neq x$

7. $M, x \models \forall \varphi$ iff $M, y \models \varphi$ for all $y \in I$

An $\mathcal{L}$-formula $\varphi$ is said to be satisfiable over $\mathbb{R}$ if there exist an assignment $h$ into $\mathbb{R}$, and a point $x \in \mathbb{R}$, such that $(\mathbb{R}, h), x=\varphi$. The formula $\varphi$ is said to be valid over $\mathbb{R}$ if $(\mathbb{R}, h), x=\varphi$ for every assignment $h$ into $\mathbb{R}$ and every $x \in \mathbb{R}$. (There is a potential ambiguity here since $(\mathbb{R},<)$ is also a Kripke frame, but in this paper we never consider Kripke semantics in $(\mathbb{R},<)$.) Clearly, $\varphi$ is valid over $\mathbb{R}$ iff $\neg \varphi$ is not satisfiable over $\mathbb{R}$. Let $L$ denote the set of $\mathcal{L}$-formulas that are valid over $\mathbb{R}$ - the logic of $\mathbb{R}$. We define $L_{\square}=L \cap \mathcal{L}_{\square}, L_{\square \forall}=L \cap \mathcal{L}_{\square \forall}$, and $\mathrm{L}_{[\partial] \forall}=\mathrm{L} \cap \mathcal{L}_{[\partial] \forall}$ - the logics of $\mathbb{R}$ in each of the respective sublanguages. Our main aim is to give simple completeness proofs for these three logics. (There is little point in considering $L$ itself, since $\square$ can be expressed with $[\partial]$.)

\section{Construction of linear models}

We now recall some well known information about lexicographic sums of monadic expansions of linear orders. Sources include $[11,18,3]$. Taken further, this becomes an extremely powerful model-theoretic method and we cite $[16,22,7,5$, 17] as further reading. 


\subsection{Lexicographic sums}

Let $\left(J,<_{J}\right)$ be a linear order, and for each $j \in J$ let $M_{j}=\left(I_{j},<_{j}, h_{j}\right)$ be a linear model. We write

$$
M=\sum_{j \in J} M_{j}
$$

for the linear model $(I,<, h)$, where $I=\left\{\langle i, j\rangle: j \in J, i \in I_{j}\right\},<$ is defined lexicographically by $\langle i, j\rangle<\left\langle i^{\prime}, j^{\prime}\right\rangle$ iff $j<_{J} j^{\prime}$ or $\left(j=j^{\prime}\right.$ and $\left.i<_{j} i^{\prime}\right)$, and $h(p)=\bigcup_{j \in J}\left(h_{j}(p) \times\{j\}\right)=\left\{\langle i, j\rangle: j \in J, i \in h_{j}(p)\right\}$ for each $p \in P V$. It can be verified that $(I,<)$ is a linear order. When $J=(\{0, \ldots, n-1\},<)$, we may write $M$ as $\sum_{j<n} M_{j}$. When $J=(\{0,1\},<)$, we may write $M$ as $M_{0}+M_{1}$. Up to isomorphism, + is associative (though not commutative), so we may omit brackets in finite sums.

For $j \in J$, we let $M\left\lceil j\right.$ denote the submodel of $M$ with domain $I_{j} \times\{j\}$. It is isomorphic to $M_{j}$ (the isomorphism is $\langle i, j\rangle \mapsto i$ ). We will sometimes identify the two, and so regard $M_{j}$ as a submodel of $M$ via this isomorphism.

\subsection{Intervals of $\mathbb{R}$}

An interval of $\mathbb{R}$ is a nonempty convex subset $X \subseteq \mathbb{R}$, regarded implicitly as a linear order $(X,<\uparrow X)$. An interval is open if it has no least element and no greatest element. We will use standard notation for intervals: $[x, y]=\{z \in$ $\mathbb{R}: x \leq z \leq y\},(x, y),[x, y)$, etc. We will be interested in linear models whose domains are (isomorphic to) intervals of $\mathbb{R}$. The following is a trivial but useful case.

DEFINITION 3.1 For $p \in P V$ we will let $\widehat{p}$ denote the one-point linear model $(\{0\}, \emptyset, h)$, where $h(p)=\{0\}$ and $h(q)=\emptyset$ for each $q \in P V \backslash\{p\}$.

EXAMPLE 3.2 Let $p, q \in P V$. Let $M_{j}=\widehat{p}$ for each $j \in \mathbb{Q}$ and $M_{j}=\widehat{q}$ for each $j \in \mathbb{R} \backslash \mathbb{Q}$. Then $\sum_{j \in \mathbb{R}} M_{j}$ is isomorphic to the linear model $M=(\mathbb{R},<, h)$, where $h(p)=\mathbb{Q}, h(q)=\mathbb{R} \backslash \mathbb{Q}$, and $h(r)=\emptyset$ for every $r \in P V \backslash\{p, q\}$.

In the example, the underlying order of $M$ was isomorphic to $\mathbb{R}$. This is an instance of a more general phenomenon:

PROPOSITION 3.3 Let $(J,<)$ be a linear order, and for each $j \in J$ let $M_{j}$ be a linear model over an interval of $\mathbb{R}$. Suppose that one of the following conditions holds:

1. $(J,<)=(\{0,1, \ldots, n\},<)$ for some integer $n \geq 0, M_{j}$ has a greatest element and no least element for each $j \in\{0,1, \ldots, n-1\}$, and $M_{n}$ has no least element and no greatest element.

2. $(J,<)=(\mathbb{Z},<)$, and for each $j \in \mathbb{Z}, M_{j}$ has a greatest element and no least element.

3. $(J,<)=(\mathbb{R},<)$, each $M_{j}$ has a least and a greatest element, and $\operatorname{dom}\left(M_{j}\right)$ is a singleton whenever $j \in \mathbb{R} \backslash \mathbb{Q}$.

Then the underlying order of $\sum_{j \in J} M_{j}$ is isomorphic to $\mathbb{R}$. 
Proof. It is well known and easily proved that a linear order is isomorphic to $\mathbb{R}$ iff it has no least element, no greatest element, is separable (has a countable dense subset), (hence) is dense, and is Dedekind complete (any nonempty subset with an upper bound has a least upper bound). It is easily checked that $\sum_{j \in J} M_{j}$ has these properties in each case.

\subsection{Shuffles}

An important and attractive type of lexicographic sum is the so-called shuffle. Shuffles give us an exceedingly simple way to define relatively complicated linear models.

Let $\mathcal{N}$ be a countable set of linear models, where each $N \in \mathcal{N}$ is based on an interval of $\mathbb{R}$ with a least element and a greatest element (such as $[0,1]$ or $\{0\}$ ). Let $N_{0}$ be a linear model based on a singleton interval. A shuffle choice map is a map $s: \mathbb{R} \rightarrow \mathcal{N} \cup\left\{N_{0}\right\}$ such that:

1. $s^{-1}(N)$ is a dense subset of $\mathbb{R}$ for each $N \in \mathcal{N}$.

2. $s(x)=N_{0}$ for each irrational $x \in \mathbb{R}$.

Since $\mathbb{Q}$ can be partitioned into infinitely many dense subsets, it is not difficult to show that shuffle choice maps exist. Choose a shuffle choice map $s$, and define

$$
M=\operatorname{Shuffle}\left(\mathcal{N} ; N_{0}\right)=\sum_{j \in \mathbb{R}} s(j) .
$$

By proposition 3.3(3), $M$ is a linear model whose underlying order is isomorphic to $\mathbb{R}$, and we will regard it as actually having $\mathbb{R}$ as its underlying order. Its formal form depends on $s$ and the isomorphism to $\mathbb{R}$, but the specific choices are immaterial here, and in any case, an argument similar to the proof that any countable dense linear order is isomorphic to $(\mathbb{Q},<)$ will show that, up to isomorphism, $M$ is independent of these choices. Whenever we use the Shuffle notation as in equation (1), we will assume that they have been tacitly chosen.

Let $M$ be the shuffle above. An element of $M$ is said to be an $M$-endpoint if it is a least or greatest element of $M \uparrow j$ for some $j \in \mathbb{R}$ (see $\S 3.1$ for the definition of $M \uparrow j)$.

LEMMA 3.4 Let $x \in M$ and $p \in \operatorname{supp}(M)$. (See §2.4 for $\operatorname{supp}(M)$.)

1. If $x$ is an $M$-endpoint, then $M, x=\langle\partial\rangle p$ and $M, x \models \diamond p$.

2. If $x$ is not an $M$-endpoint, suppose that $x \in M \uparrow j$ for (unique) $j \in \mathbb{R}$. Then $M, x \models \diamond p$ iff $M \uparrow j, x \models \diamond p$, and $M, x \models\langle\partial\rangle p$ iff $M \uparrow j, x \mid\langle\partial\rangle p$.

3. There are $y, z \in \mathbb{R}$ with $y<x<z, M, y \models p$, and $M, z \models p$.

Proof. [Proof sketch] For part 1, suppose that $x$ is the greatest point of $M \uparrow j$, for some $j \in \mathbb{R}$. Let $y>x$ be given. Plainly, $y \in M \uparrow k$ for some $k \in \mathbb{R}$ with $k>j$. Pick $N \in \mathcal{N} \cup\left\{N_{0}\right\}$ and $t \in N$ with $N, t \models p$. As $s^{-1}(N)$ is dense in $\mathbb{R}$, we may find $l \in(j, k)$ with $s(l)=N$, so $M \uparrow l \cong N$. Let $z$ be the element of $M \uparrow l$ corresponding to $t$ under this isomorphism. Then $M, z \models p$ and $z \in(x, y)$. Since $y$ was arbitrary, $M, x \models\langle\partial\rangle p \wedge \diamond p$. The case where $x$ is the least point of $M \uparrow j$ is similar. Part 2 holds simply because the part of 
$M\lceil j$ excluding its endpoints is an open interval of $\mathbb{R}$ containing $x$. Finally, part 3 holds because for each $N \in \mathcal{N} \cup\left\{N_{0}\right\}$ there are arbitrarily large and small $j \in \mathbb{R}$ with $s(j)=N$.

EXAMPLE 3.5 Let $p, q \in P V$.

1. Shuffle $(\{\widehat{p}\} ; \widehat{q})$ is, up to isomorphism, the model $M$ of example 3.2 .

2. Let $N=\widehat{p}+\operatorname{Shuffle}(\emptyset ; \widehat{p})+\widehat{p}$. This is a linear model whose underlying order is isomorphic to $[0,1]$, and all its points satisfy $p$ and only $p$.

3. $S=\operatorname{Shuffle}(\{N\} ; \widehat{q})$ is a linear model that can be described up to isomorphism as: each rational in $\mathbb{R}$ is replaced by a non-singleton closed interval of $\mathbb{R}$ whose points satisfy only $p$, and each irrational is left intact and made to satisfy only $q$. The $S$-endpoints are the endpoints of the closed intervals and the intact irrationals. The endpoints satisfy $\langle\partial\rangle p \wedge\langle\partial\rangle q$, while the other points satisfy $\square(p \wedge \neg q)$. The underlying order of $S$ is isomorphic to $\mathbb{R}$.

4. Shuffle $(\{N, \widehat{p}\} ; \widehat{q})$ is rather different: again up to isomorphism, we split $\mathbb{Q}$ into two dense subsets, replace the points of the first set by copies of $N$, make the points of the second set satisfy only $p$, and make the irrationals satisfy only $q$ as before. This model is not isomorphic to $S$ above, because it has singleton subintervals (endpoints) satisfying $p$ that are not part of any longer interval whose points satisfy $p$. However, perhaps surprisingly, an Ehrenfeucht-Fraïssé game will show that it is indistinguishable from $S$ in $\mathcal{L}$.

Armed with these devices and standard modal methods, we will be able to prove completeness theorems for $\mathbb{R}$ really rather easily. There are two main steps. First, given an appropriate finite Kripke frame $(W, R)$, by applying shuffle we obtain a linear model whose domain is isomorphic to $\mathbb{R}$, and which gives rise to a certain function $f: \mathbb{R} \rightarrow W$. Second, given an assignment, say $g$, into $(W, R)$, we define a new assignment $h=f^{-1} \circ g$ into $\mathbb{R}$, yielding a linear model $M=(\mathbb{R},<, h)$. From these two main steps we can prove the following 'satisfaction' lemma: $M, x \models \varphi$ iff $(W, R, g), f(x) \models \varphi$, for each $x \in \mathbb{R}$ and $\mathcal{L}$-formula $\varphi$ in the appropriate fragment. The finite model property for each of the logics under consideration yields a finite model $(W, R, g)$ satisfying any given consistent formula $\varphi$, which is turned into a linear model satisfying $\varphi$ as above.

\section{The logic of $\mathbb{R}$ with}

We start with the classical result of McKinsey and Tarski [13] that the logic of $\square$ over $\mathbb{R}$ is $\mathrm{S} 4$. So in this section we work with the language $\mathcal{L}_{\square}$ whose formulas involve only $\top$, atoms, the boolean operations, and $\square$. Recall that $S 4$ is the smallest set of $\mathcal{L}_{\square}$-formulas that contains the axioms:

1. all propositional tautologies

2. $\square(p \rightarrow q) \rightarrow(\square p \rightarrow \square q)$

normality 
3. $\square p \rightarrow p$

reflexivity

4. $\square p \rightarrow \square \square p$

transitivity

and is closed under the inference rules:

1. modus ponens: $\frac{\varphi, \varphi \rightarrow \psi}{\psi}$

2. generalisation (or necessitation) for $\square$ : $\frac{\varphi}{\square \varphi}$

3. substitution: $\frac{\varphi(p)}{\varphi(\psi / p)}$

Let $(W, R)$ be a finite Kripke frame in which all the axioms of $\mathrm{S} 4$ are valid, and such that $W \subseteq P V$ (this will allow us to define models $\widehat{w}$ and write $\mathcal{L}$ formulas such as $\diamond w$, for $w \in W)$. It follows that $R$ is reflexive and transitive. Define a binary relation $R^{\bullet}$ on $W$ by $R^{\bullet} w u$ iff $R w u \wedge \neg R u w$. For each $w \in W$ define $C(w)=\{u \in W: R w u \wedge R u w\}$.

DEFINITION 4.1 For each $w \in W$, we define a linear model $N_{w}$ over $\mathbb{R}$ by complete induction on $|R(w)|$ :

$$
N_{w}=\operatorname{Shuffle}\left(\left\{\widehat{w}+N_{u}+\widehat{w}: u \in R^{\bullet}(w)\right\} \cup\{\widehat{u}: u \in C(w)\} ; \widehat{w}\right) .
$$

We check that $N_{w}$ is well defined. If $u \in R^{\bullet}(w)$ then $R(u) \subseteq R(w)$ by transitivity of $R$, and plainly, $w \in R(w) \backslash R(u)$. So $|R(u)|<|R(w)|$. Inductively, $N_{u}$ is a well defined linear model over $\mathbb{R}$. So the underlying order of $\widehat{w}+N_{u}+\widehat{w}$ is isomorphic to an interval of $\mathbb{R}$ with a least and a greatest element. All models $\widehat{u}, \widehat{w}$ are based on singleton intervals, so $N_{w}$ is a legal shuffle and a well defined linear model over $\mathbb{R}$.

Since $N_{w}$ is 'made' wholly from linear models of the form $\widehat{u}$ for $u \in R(w)$, we have $\operatorname{supp}\left(N_{w}\right) \subseteq R(w)$, and for each $x \in \mathbb{R}$ there is a unique $u \in R(w)$ with $N_{w}, x \models u$. (This can be proved formally by a trivial induction on $|R(w)|$.) We write $f_{w}(x)$ for this $u$. So we have defined a map $f_{w}: \mathbb{R} \rightarrow W$. For all $x \in \mathbb{R}$ and $u \in W$,

$$
N_{w}, x=u \Longleftrightarrow f_{w}(x)=u \text {. }
$$

LEMMA 4.2 For every $w \in W$, the following hold:

1. We have $\operatorname{rng}\left(f_{w}\right)=\operatorname{supp}\left(N_{w}\right)=R(w)$.

2. For each $v \in W$ and $x \in \mathbb{R}$ we have $N_{w}, x=\diamond v$ iff $R\left(f_{w}(x), v\right)$.

Proof. By induction on $|R(w)|$. Assume the lemma inductively for every $u \in$ $W$ with $|R(u)|<|R(w)|$ - in particular, every $u \in R^{\bullet}(w)$. By definition, $\operatorname{supp}\left(N_{w}\right)=\left\{u \in P V: \exists x \in \mathbb{R}\left(N_{w}, x \models u\right)\right\}$. By the above, this is $\{u \in W$ : $\left.\exists x \in \mathbb{R}\left(u=f_{w}(x)\right)\right\}=\operatorname{rng}\left(f_{w}\right)$. Noting that $\operatorname{supp}(\widehat{u})=\{u\}$, it follows from the definition of $N_{w}$ that $\operatorname{supp}\left(N_{w}\right)=\bigcup\left\{\{w\} \cup \operatorname{supp}\left(N_{u}\right): u \in R^{\bullet}(w)\right\} \cup C(w)$. Inductively, this is $\bigcup\left\{\{w\} \cup R(u): u \in R^{\bullet}(w)\right\} \cup C(w)=R(w)$. This proves part 1. For part 2, let $v, x$ be given. There are two cases. 
Case 1. If $x$ is an $N_{w}$-endpoint, then inspection of the definition of $N_{w}$ shows that $f_{w}(x)=w$ or $f_{w}(x) \in C(w)$. Either way, $f_{w}(x) \in C(w)$. So by transitivity of $R$ we have $R\left(f_{w}(x)\right)=R(w)$, and hence $R\left(f_{w}(x), v\right)$ iff $v \in R(w)$. By part 1 , $R(w)=\operatorname{supp}\left(N_{w}\right)$. Also, $v \in \operatorname{supp}\left(N_{w}\right)$ iff $N_{w}, x=\diamond v(\Rightarrow$ is by lemma 3.4, and $\Leftarrow$ is trivial). Stringing all this together, we see that $R\left(f_{w}(x), v\right)$ iff $N_{w}, x \models \diamond v$.

Case 2. If not, then $x \in N_{w} \uparrow j \cong \widehat{w}+N_{u}+\widehat{w}$ for some $j \in \mathbb{R}$ and $u \in R \bullet(w)$. We identify $N_{u}$ with the submodel of $N_{w}\left\lceil j \subseteq N_{w}\right.$ as usual. As $x$ is not a $N_{w^{-}}$ endpoint, we have $x \in N_{u}$. By lemma 3.4(2), $N_{w}, x=\diamond v$ iff $N_{w}\lceil j, x=\diamond v$. The least and greatest points of $N_{w}\left\lceil j\right.$ do not affect $\diamond$, so this is iff $N_{u}, x \models \diamond v$. Inductively, this is iff $R\left(f_{u}(x), v\right)$. But plainly, $f_{u}(x)=f_{w}(x)$.

Now fix $w \in W$, and write $N_{w}$ and $f_{w}$ simply as $N$ and $f$, respectively. Let $g$ : $P V \rightarrow \wp(W)$ be an assignment and let $\mathcal{M}$ be the Kripke model $(W, R, g)$. Define an assignment $h: P V \rightarrow \wp(\mathbb{R})$ by $h(p)=f^{-1}(g(p))=\{x \in \mathbb{R}: \mathcal{M}, f(x) \models p\}$, for each $p \in P V$. Let $M$ be the linear model $(\mathbb{R},<, h)$. Of course, $M$ depends on $\mathcal{M}$ and $w$. We now have two linear models $N, M$ over $\mathbb{R}$ and we will use them both below.

LEMMA 4.3 For every $\psi \in \mathcal{L}_{\square}$ and $x \in \mathbb{R}$, we have $M, x \models \psi$ iff $\mathcal{M}, f(x) \models$ $\psi$.

Proof. By induction on $\psi$. The atomic and boolean cases are easy and we omit them. Assume the lemma for $\psi$, and consider $\diamond \psi$. First suppose that $\mathcal{M}, f(x) \models \diamond \psi$, so there is $u \in W$ with $R(f(x), u)$ and $\mathcal{M}, u \mid=\psi$. By lemma 4.2, $N, x \models \diamond u$. Inductively, every $y \in \mathbb{R}$ with $N, y \models u$ satisfies $M, y \models \psi$ (since $f(y)=u)$. It follows that $M, x \models \diamond \psi$.

Conversely, suppose that $M, x \models \diamond \psi$. We claim that for some $u \in W$, every open interval of $\mathbb{R}$ containing $x$ contains a point $y$ with $M, y \models \psi$ and $f(y)=u$. For if not, for each $u$ there is an open interval $O_{u}$ containing $x$ but containing no such point $y$. Let $O=\bigcap_{u \in W} O_{u}$. Since $W$ is finite, $O$ is again an open interval containing $x$. But $M, x \models \diamond \psi$, so $O$ contains a point $y$ with $M, y \models \psi$. Let $f(y)=u$. Then $y \in O_{u}$, contradicting the definition of $O_{u}$. This proves the claim.

Let $u$ be as in the claim. Plainly, $N, x=\diamond u$, so by lemma $4.2, R(f(x), u)$. Also, inductively we have $\mathcal{M}, u \models \psi$. Hence, $\mathcal{M}, f(x) \models \diamond \psi$ as required.

THEOREM 4.4 (McKinsey-Tarski, 1944) The $\mathcal{L}_{\square}$-logic $\mathrm{L}_{\square}$ of $\mathbb{R}$ is $\mathrm{S} 4$.

Proof. It is easy to check that the $\mathrm{S} 4$ axioms are valid over $\mathbb{R}$, and that the inference rules preserve validity. So $\mathrm{S} 4 \subseteq \mathrm{L}_{\square}$. For the converse, take $\varphi \in \mathcal{L}_{\square}$ with $\varphi \notin \mathrm{S} 4$. We will show that $\neg \varphi$ is satisfiable over $\mathbb{R}$, so that $\varphi \notin \mathrm{L}_{\square}$, completing the proof.

It is known that $\mathrm{S} 4$ has the finite model property. (This can be proved by filtration: see, e.g., [4, corollary 5.32].) So $\neg \varphi$ is satisfied in a finite Kripke model $\mathcal{M}=(W, R, g)$ such that all the $\mathrm{S} 4$-axioms are valid in the Kripke frame $(W, R)$. It is immaterial what the elements of $W$ are, so we may assume without loss of generality that $W \subseteq P V$. Choose $w \in W$ such that $\mathcal{M}, w \mid=\neg \varphi$.

We now suppose that $W, R, \mathcal{M}$, and $w$ are as in the foregoing discussion. This can be done without loss of generality. Define $f$ and $M$ as above. By lemma $4.2(1)$, there is $x \in \mathbb{R}$ with $f(x)=w$. As $\mathcal{M}, w \models \neg \varphi$, lemma 4.3 yields $M, x=\neg \varphi$. So $\neg \varphi$ is satisfiable over $\mathbb{R}$ and $\varphi \notin \mathrm{L}_{\square}$ as required. 
The map $f$ is an interior map, as in several other proofs of this result. It is worth noting that the proof transforms a finite Kripke model satisfying a formula effectively into an explicit and simple description of a model over $\mathbb{R}$ that satisfies the formula. It is easy to write down the description in practice, using shuffles.

EXAMPLE 4.5 The formula $\varphi=p \wedge \diamond(\neg p \wedge \diamond p)$ is plainly true at 0 in the Kripke model $\mathcal{M}=(\{0,1,2\}, \leq, g)$ where $g(p)=\{0,2\}$. We assume as above that $0,1,2 \in P V$, and construct three linear models:

$$
\begin{aligned}
& N_{2}=\operatorname{Shuffle}(\{\widehat{2}\} ; \widehat{2}) \\
& N_{1}=\operatorname{Shuffle}\left(\left\{\widehat{1}+N_{2}+\widehat{1}, \widehat{1}\right\} ; \widehat{1}\right) \\
& N_{0}=\operatorname{Shuffle}\left(\left\{\widehat{0}+N_{1}+\widehat{0}, \widehat{0}+N_{2}+\widehat{0}, \widehat{0}\right\} ; \widehat{0}\right)
\end{aligned}
$$

The underlying order of $N_{0}$ is $\mathbb{R}$. We define $f=f_{0}: \mathbb{R} \rightarrow\{0,1,2\}$ as above. So for each $i \in\{0,1,2\}, f^{-1}(i)$ is the set of points of $N_{0}$ lying in 'copies' of $\widehat{i}$. We define $h: P V \rightarrow \wp(\mathbb{R})$ by $h(q)=f^{-1}(g(q))$, for $q \in P V$. So $h(p)=$ $f^{-1}(0) \cup f^{-1}(2)$. We define $M$ to be the linear model $(\mathbb{R},<, h)$. Then $M, x=\varphi$ for any $x$ in a copy of $\widehat{0}$. Indeed it is plain that any such $x$ satisfies $p$ and has arbitrarily close to it points $y$ in copies of $\widehat{1}$. Such $y$ satisfy $\neg p$, and have points $z$ in copies of $\widehat{2}$ arbitrarily close to them; such $z$ satisfy $p$.

\section{$5 \quad$ The logic of $\mathbb{R}$ with $\square$ and $\forall$}

We now move on to the language $\mathcal{L}_{\square \forall}$ containing formulas using both $\square$ and $\forall$. In [20], Shehtman showed that the logic of $\mathbb{R}$ in this language is S4UC. The logic S4UC is the smallest set of $\mathcal{L}_{\square \forall}$-formulas closed under the inference rules of modus ponens, generalisation for both $\square$ and $\forall$, and substitution, and containing the following axioms:

1. all propositional tautologies

2. $\square(p \rightarrow q) \rightarrow(\square p \rightarrow \square q)$ normality

3. $\square p \rightarrow p$ reflexivity

4. $\square p \rightarrow \square \square p$ transitivity

5. $\forall(p \rightarrow q) \rightarrow(\forall p \rightarrow \forall q)$ normality

6. $\forall p \rightarrow p$ reflexivity

7. $\forall p \rightarrow \forall \forall p$ transitivity

8. $\exists \forall p \rightarrow p$ symmetry

9. $\forall p \rightarrow \square p$ 'U'

10. $\forall(\square p \vee \square \neg p) \rightarrow \forall p \vee \forall \neg p$

connectedness, ' $\mathrm{C}$ '

Let $(W, R)$ be a finite Kripke frame in which all the axioms of S4UC are valid and such that $W \subseteq P V$. So $R$ is reflexive and transitive. We will apply the same idea as in the preceding section, but since $\forall$ is in the language, we need to arrange that the map $f: \mathbb{R} \rightarrow W$ is surjective. To do this, we will use that $(W, R)$ is connected. 
DEFINITION 5.1 Let $\mathcal{F}=(W, R)$ be a Kripke frame. A connected component of $\mathcal{F}$ is a minimal nonempty subset $D \subseteq W$ such that for all $w \in W$ :

- if $w \in D$ then $R(w) \subseteq D$

- if $w \in W \backslash D$ then $R(w) \subseteq W \backslash D$

If $k$ is an integer, $\mathcal{F}$ is said to be $k$-connected if it has at most $k$ connected components, and connected if it is 1-connected. A Kripke model is said to be connected or $k$-connected if its frame has this property.

The slight differences of definition 5.1 from definitions in [20, 12] will not matter, since we will not formally use any results involving connectedness from those papers.

Indeed, $(W, R)$ is connected. This is easy to see (cf. [20, lemma 8]). For if $D$ is a connected component of $(W, R)$, let $g$ be an assignment into $(W, R)$ with $g(p)=D$, and let $w \in W$. Then $(W, R, g), w=\forall(\square p \vee \square \neg p)$. Axiom $\mathrm{C}$ is valid in $(W, R)$, so $(W, R, g), w \models \forall p \vee \forall \neg p$, and hence $D=W$ or $D=\emptyset$. Since $D$ is nonempty, $D=W$.

As $(W, R)$ is finite and connected, a little thought shows that there exist points $d_{0}, u_{0}, d_{1}, u_{1}, \ldots, d_{n-1}, u_{n-1}, d_{n} \in W$, for some finite $n$, such that:

- $R u_{j} d_{j}$ and $R u_{j} d_{j+1}$ for each $j<n$

- $W=\bigcup_{j<n} R\left(u_{j}\right)$

For each $w \in W$, let $N_{w}$ be the linear model of definition 4.1, with underlying order $\mathbb{R}$.

LEMMA 5.2 For each $x \in \mathbb{R}$ and $u \in W$, we have $u \in \operatorname{supp}\left(N_{w}\right)$ iff there are $y, z \in \mathbb{R}$ with $y<x<z, N_{w}, y \models u$, and $N_{w}, z \models u$.

Proof. $\Rightarrow$ is immediate from lemma $3.4(3)$, and $\Leftarrow$ is trivial.

Now define

$$
N=\left(\sum_{j<n}\left(N_{d_{j}}+\widehat{u_{j}}+N_{u_{j}}+\widehat{u_{j}}\right)\right)+N_{d_{n}} .
$$

In effect, $N$ is the finite sum

$$
N_{d_{0}}+\widehat{u_{0}}+N_{u_{0}}+\widehat{u_{0}}+N_{d_{1}}+\widehat{u_{1}}+N_{u_{1}}+\widehat{u_{1}}+\cdots+N_{u_{n-1}}+\widehat{u_{n-1}}+N_{d_{n}} .
$$

A couple of applications of proposition 3.3(1) show that $N$ is a linear model whose underlying linear order is isomorphic to $\mathbb{R}$. As usual, we will assume that its underlying order is actually $\mathbb{R}$, and that each of the $N_{d_{j}}, N_{u_{j}}$, and two copies of $\widehat{u_{j}}$ are submodels of $N$.

As in $\S 4$, for each $x \in \mathbb{R}$ there is a unique $u \in W$ with $N, x=u$, and we write $f(x)$ for this $u$. Thus, $f: \mathbb{R} \rightarrow W$. By lemma 4.2(1) and the choice of $u_{j}$, we have

$$
\operatorname{rng}(f)=\operatorname{supp}(N) \supseteq \bigcup_{j<n} \operatorname{supp}\left(N_{u_{j}}\right)=\bigcup_{j<n} R\left(u_{j}\right)=W .
$$

So $f$ is surjective. 
LEMMA 5.3 Let $x \in \mathbb{R}$ and $w \in W$. Then $N, x \models \diamond w$ iff $R(f(x), w)$.

Proof. If $x \in N_{d_{j}}$ for some $j \leq n$ or $x \in N_{u_{j}}$ for some $j<n$, the result follows from lemma 4.2(2), since these submodels are based on open intervals of $\mathbb{R}$. Suppose for some $j<n$ that $x$ is in the submodel $\widehat{u_{j}}$ that is preceded by $N_{d_{j}}$ and followed by $N_{u_{j}}$. Clearly, $N, x \models \diamond w$ iff (a) arbitrarily large elements of $N_{d_{j}}$ satisfy $w$, or (b) $N, x \models w$, or (c) arbitrarily small elements of $N_{u_{j}}$ satisfy $w$. Now by lemmas 5.2 and 4.2(1), (a) holds iff $w \in \operatorname{supp}\left(N_{d_{j}}\right)=R\left(d_{j}\right)$, and (c) holds iff $w \in \operatorname{supp}\left(N_{u_{j}}\right)=R\left(u_{j}\right)$. Plainly, (b) holds iff $w=u_{j}$. So $N, x \models \diamond w$ iff $w \in R\left(d_{j}\right) \cup\left\{u_{j}\right\} \cup R\left(u_{j}\right)$. Because $R$ is reflexive and transitive and $R u_{j} d_{j}$, we have $R\left(d_{j}\right) \cup\left\{u_{j}\right\} \cup R\left(u_{j}\right)=R\left(u_{j}\right)=R(f(x))$. So $N, x=\diamond w$ iff $R(f(x), w)$, as required. The argument when $x$ is in the submodel $\widehat{u_{j}}$ between $N_{u_{j}}$ and $N_{d_{j+1}}$ is similar, using that $R u_{j} d_{j+1}$.

Now, as before, let $g: P V \rightarrow \wp(W)$ be an assignment into $W$, and let $\mathcal{M}$ be the Kripke model $(W, R, g)$. Define the linear model $M=(\mathbb{R},<, h)$, where $h(p)=f^{-1}(g(p))$ for each $p \in P V$.

LEMMA 5.4 For every $\psi \in \mathcal{L}_{\square \forall}$ and $x \in \mathbb{R}$, we have $M, x \models \psi$ iff $\mathcal{M}, f(x) \models$ $\psi$.

Proof. The proof is the same as for lemma 4.3, but there is an additional case: $\forall \psi$. So assume the lemma inductively for $\psi$, and let $x \in \mathbb{R}$ be given. If $\mathcal{M}, f(x) \models \forall \psi$, then $\mathcal{M}, w \models \psi$ for all $w \in W$. Inductively, $M, y \models \psi$ for all $y \in \mathbb{R}$, and we obtain $M, x \models \forall \psi$. Conversely, assume that $M, x \models \forall \psi$, and let $w \in W$ be given. As $f$ is surjective, we can find $y \in \mathbb{R}$ with $f(y)=w$. By assumption, $M, y \models \psi$, and inductively, $\mathcal{M}, f(y) \models \psi$ as well. Since $w$ was arbitrary, we obtain $\mathcal{M}, f(x) \models \forall \psi$ as required.

THEOREM 5.5 (Shehtman, 1999) The $\mathcal{L}_{\square \forall-l o g i c ~} \mathrm{~L}_{\square \forall}$ of $\mathbb{R}$ is S4UC.

Proof. Again it is easy to check soundness: that $\mathrm{S} 4 \mathrm{UC} \subseteq \mathrm{L}_{\square \forall}$. (Axiom C is valid over $\mathbb{R}$ because $\mathbb{R}$ is connected: it cannot be written as the union of two disjoint nonempty open sets.) For the converse, we take a formula $\varphi \notin$ S4UC and show that $\neg \varphi$ is satisfiable over $\mathbb{R}$, so that $\varphi \notin \mathrm{L} \square \forall$.

By [20, theorem 10] (proved by filtration), S4UC has the finite model property. So we may take a finite Kripke model $\mathcal{M}=(W, R, g)$ satisfying $\neg \varphi$ and in whose frame $(W, R)$ all axioms of S4UC are valid. We may assume that $W, R, g$ are the same as above. Define $f, M$ as above. Take $w \in W$ with $\mathcal{M}, w \models \neg \varphi$. As $f$ is surjective, we may find $x \in \mathbb{R}$ with $f(x)=w$. By lemma 5.4, $M, x \models \neg \varphi$. Thus, $\neg \varphi$ is satisfiable over $\mathbb{R}$, which completes the proof.

\section{The logic of $\mathbb{R}$ with $[\partial]$ and $\forall$}

Finally we consider the language $\mathcal{L}_{[\partial] \forall}$ containing formulas using $[\partial]$ and $\forall$ but not $\square$. Actually we will use $\square$, but as an abbreviation: $\square \varphi$ will abbreviate $\varphi \wedge[\partial] \varphi$. (It can be checked that the semantics of $\square$ in linear models - though not in Kripke models - is as in earlier sections.)

The logic of $\mathbb{R}$ in the language $\mathcal{L}_{[\partial]}$ is $\mathrm{KD}_{4} \mathrm{G}_{2}$ - this was conjectured by Shehtman [19] and proved by Shehtman [21] and Lucero-Bryan [12, theorem 
4.5]. Lucero-Bryan goes on to show [12, corollary 5.27] that the logic of $\mathbb{R}$ in the language $\mathcal{L}_{[\partial] \forall}$ is $\mathrm{KD} 4 \mathrm{G}_{2} . \mathrm{UC}$. The logic $\mathrm{KD}_{4} \mathrm{G}_{2} . \mathrm{UC}$ is the smallest set of

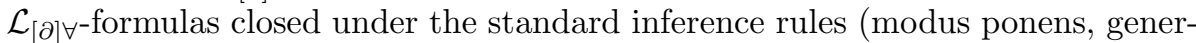
alisation for $[\partial]$ and $\forall$, and substitution) and containing the following axioms:

1. all propositional tautologies

2. $[\partial](p \rightarrow q) \rightarrow([\partial] p \rightarrow[\partial] q)$ normality

3. $\langle\partial\rangle \top$ seriality

4. $[\partial] p \rightarrow[\partial][\partial] p$ transitivity

5. $\forall(p \rightarrow q) \rightarrow(\forall p \rightarrow \forall q)$ normality

6. $\forall p \rightarrow p$ reflexivity

7. $\forall p \rightarrow \forall \forall p$ transitivity

8. $\exists \forall p \rightarrow p$ symmetry

9. $\forall p \rightarrow[\partial] p$ 'U'

10. $\forall(\square p \vee \square \neg p) \rightarrow \forall p \vee \forall \neg p$ connectedness, ' $\mathrm{C}$ '

11. $[\partial]\left(\bigvee_{0 \leq i \leq 2} \square \varphi_{i}\right) \rightarrow \bigvee_{0 \leq i \leq 2}[\partial] \neg \varphi_{i}$ where $p_{0}, p_{1}, p_{2} \in P V$ and $\varphi_{i}=p_{i} \wedge \bigwedge\left\{\neg p_{j}: 0 \leq j \leq 2, j \neq i\right\}$ for each $i \in\{0,1,2\}$

The logic $\mathrm{KD}_{4} \mathrm{G}_{2}$ is defined analogously in the language $\mathcal{L}_{[\partial]}$ by deleting axioms 5-10.

Let $\mathcal{F}=(W, R)$ be a finite Kripke frame in which all axioms of $\mathrm{KD}_{4} \mathrm{G}_{2}$.UC are valid, and with $W \subseteq P V$. Importantly, $R$ may not be reflexive. But we do have:

- $R$ is transitive.

- $R(w) \neq \emptyset$ for every $w \in W$.

- $\mathcal{F}$ is connected (using axiom $\mathrm{C}$ ).

- $\mathcal{F}$ is 'locally 2-connected'. That is, for every $w \in W$, the frame $(R(w), R \uparrow$ $R(w)$ ) is 2-connected (see definition 5.1) and so has at most two connected components. (This is easy to prove using validity of $\mathrm{G}_{2}$ in $\mathcal{F}$.)

As earlier, define the binary relation $R^{\bullet}$ on $W$ by $R^{\bullet} w u$ iff $R w u \wedge \neg R u w$. For $w \in W$, define $C(w)=\{u \in W: R w u \wedge R u w\}$. We say that $w$ is a leaf if $R^{\bullet}(w)=\emptyset$. In that case, the axiom $\langle\partial\rangle \top$ and transitivity give $R w w$. For $w \in W$ we define $W_{w}=R(w) \cup\{w\}$, and define the Kripke frame

$$
\mathcal{F}_{w}=\left(W_{w}, R \uparrow W_{w}\right) .
$$

This is connected - a connected component containing $w$ must be $W_{w}$ - and a generated subframe of $\mathcal{F}$.

LEMMA 6.1 For each connected generated subframe $\mathcal{G}$ of $\mathcal{F}$, there is a linear model $\overline{\mathcal{G}}$ based on $\mathbb{R}$, and such that for each $x \in \mathbb{R}$ and $v \in \mathcal{G}$ : 
G1. There is a unique $u \in P V$ with $\overline{\mathcal{G}}, x \models u$. Moreover, $u \in \mathcal{G}$. We will write this $u$ as $f_{\mathcal{G}}(x)$.

G2. $\overline{\mathcal{G}}, x \models\langle\partial\rangle v$ iff $R\left(f_{\mathcal{G}}(x), v\right)$.

G3. There are $y<x<z$ in $\mathbb{R}$ with $\overline{\mathcal{G}}, y \models v$ and $\overline{\mathcal{G}}, z \models v$.

G4. If $v$ is a leaf, there are linear models $A, B$ such that $\overline{\mathcal{G}} \cong A+\overline{\mathcal{F}_{v}}+B$. (Reminder: linear models are nonempty.)

Proof. We prove the lemma by complete induction on $|\mathcal{G}|$. So take a connected generated subframe $\mathcal{G}$ of $\mathcal{F}$, and inductively assume the lemma for smaller subframes than $\mathcal{G}$. There are two cases.

Case 1. Suppose that $\mathcal{G}=\mathcal{F}_{w}$ for some reflexive $w \in W$ (i.e., with $R w w$ ). Choose such a $w$ (it need not be unique). Now, as in definition 4.1, we let

$$
\overline{\mathcal{G}}=\operatorname{Shuffle}\left(\left\{\widehat{w}+\overline{\mathcal{F}_{u}}+\widehat{w}: u \in R^{\bullet}(w)\right\} \cup\{\widehat{u}: u \in C(w)\} ; \widehat{w}\right)
$$

Inductively, $\overline{\mathcal{F}_{u}}$ is defined for each $u \in R^{\bullet}(w)$, so as earlier, we see that this shuffle is well defined. It is easy to confirm that $\overline{\mathcal{G}}$ meets the requirements G1-G4. We leave the reader to verify G1 and G3. We briefly check G2. It holds inductively for any $x$ in a submodel of $\overline{\mathcal{G}}$ of the form $\overline{\mathcal{F}}$. Any $x$ not in such a submodel is a $\overline{\mathcal{G}}$-endpoint, so $\overline{\mathcal{G}}, x \models\langle\partial\rangle v$ iff $v \in \operatorname{supp}(\overline{\mathcal{G}})(\Rightarrow$ is trivial and $\Leftarrow$ follows from lemma 3.4(1)). It follows easily from G1 and G3 that $\operatorname{supp}(\overline{\mathcal{G}})=W_{w}$, so this is iff $v \in W_{w}$. Since $w$ is reflexive, this is iff $R(w, v)$. But $f_{\mathcal{G}}(x) \in C(w)$, so by transitivity of $R$, this is iff $R\left(f_{\mathcal{G}}(x), v\right)$, as required.

We now check G4. Suppose that $v \in \mathcal{G}=\mathcal{F}_{w}$ is a leaf. If $R^{\bullet} w v$ then it is plain that G4 holds for $v$, since $\overline{\mathcal{F}_{v}}$ is an 'ingredient' of the shuffle in equation (4) defining $\overline{\mathcal{G}}$. If instead $\neg R^{\bullet} w v$, then $v \in C(w)$, so $\mathcal{F}_{v}=\mathcal{F}_{w}=\mathcal{G}$. Since $\overline{\mathcal{G}}$ is a shuffle, it is easily seen that $\overline{\mathcal{G}} \cong A+\overline{\mathcal{G}}+B=A+\overline{\mathcal{F}_{v}}+B$ for some $A, B$.

Case 2. Suppose otherwise. As $\mathcal{G}$ is connected and locally 2-connected, a little thought shows that there is a sequence

$$
\ldots u_{-1}, d_{0}, u_{0}, d_{1}, u_{1}, d_{2}, \ldots
$$

of elements of $\mathcal{G}$ with the following properties:

- $R u_{i} d_{i} \wedge R u_{i} d_{i+1}$ for each $i \in \mathbb{Z}$.

- $\mathcal{G}=\bigcup_{j<i} W_{u_{j}}=\bigcup_{k>i} W_{u_{k}}$ for each $i \in \mathbb{Z}$.

- $\left\{d_{i}: i \in \mathbb{Z}\right\}$ is the set of leaves that lie in $\mathcal{G}$.

- For each $i \in \mathbb{Z}$, let $C_{i}$ and $D_{i}$ be the connected components of the frame $\left(R\left(u_{i}\right), R \uparrow R\left(u_{i}\right)\right)$ that contain $d_{i}$ and $d_{i+1}$, respectively. Then $R\left(u_{i}\right)=$ $C_{i} \cup D_{i}$.

We briefly indicate one way to choose such a sequence. As $\mathcal{G}$ is connected, there is a finite 'zigzag cycle' $d_{0}, u_{0}, \ldots, d_{n-1}, u_{n-1}, d_{n}$ with $d_{n}=d_{0}, R u_{i} d_{i} \wedge R u_{i} d_{i+1}$ for each $i<n$, every leaf in $\mathcal{G}$ is among $d_{0}, \ldots, d_{n}$, and $\mathcal{G}=\bigcup_{i<n} W_{u_{i}}$. For each $d_{i}$ that is not a leaf, there is a leaf $d_{i}^{\prime}$ in $\mathcal{G}$ with $R d_{i} d_{i}^{\prime}$. We can replace $d_{i}$ by $d_{i}^{\prime}$. 
So we may assume that all the $d_{i}$ are leaves. Take any $i<n$. As $\left(R\left(u_{i}\right), R \uparrow\right.$ $R\left(u_{i}\right)$ ) is 2-connected, it has connected components $C, D$ (possibly equal) with $C \cup D=R\left(u_{i}\right)$. Suppose $d_{i} \in C$, say. If $d_{i+1} \notin D$ then choose any leaf $d \in D$ and replace the part $d_{i}, u_{i}, d_{i+1}$ of the cycle by $d_{i}, u_{i}, d, u_{i}, d_{i+1}$. Do this for each $i<n$. After these insertions we obtain a cycle $d_{0}, u_{0}, \ldots, d_{m-1}, u_{m-1}, d_{m}$ with $d_{m}=d_{0}$, for some $m \geq n$. Now the $\mathbb{Z}$-sequence

$$
\ldots, u_{m-1}, d_{0}, u_{0}, \ldots, d_{m-1}, u_{m-1}, d_{0}, u_{0}, \ldots, d_{m-1}, u_{m-1}, d_{0}, \ldots
$$

has the required properties.

Each $C_{i}(i \in \mathbb{Z})$ is the domain of a connected generated subframe of $\mathcal{F}$ which we denote by $\mathcal{C}_{i}$, and similarly for $D_{i}$. If $\mathcal{C}_{i}=\mathcal{G}$, then $u_{i} \in \mathcal{G}=\mathcal{C}_{i} \subseteq R\left(u_{i}\right) \subseteq$ $W_{u_{i}} \subseteq \mathcal{G}$, so $u_{i}$ is reflexive and $\mathcal{G}=\mathcal{F}_{u_{i}}$, contradicting the case assumption. So $\left|\mathcal{C}_{i}\right|<|\mathcal{G}|$, and similarly, $\left|\mathcal{D}_{i}\right|<|\mathcal{G}|$. Let $\overline{\mathcal{C}_{i}}, \overline{\mathcal{D}_{i}}$ be the linear models given by the inductive hypothesis. As $d_{i} \in C_{i}, d_{i+1} \in D_{i}$, and they are leaves, by the inductive hypothesis there are linear models $A_{i}, B_{i}, A_{i}^{\prime}, B_{i}^{\prime}(i \in \mathbb{Z})$ such that:

$$
\begin{aligned}
& \overline{\mathcal{C}_{i}} \cong A_{i}+\overline{\mathcal{F}_{d_{i}}}+B_{i} \\
& \overline{\mathcal{D}_{i}} \cong A_{i}^{\prime}+\overline{\mathcal{F}_{d_{i+1}}}+B_{i}^{\prime}
\end{aligned}
$$

Plainly, $A_{i}$ has a greatest element and no least element, $B_{i}$ has a least element and no greatest element, and similarly for $A_{i}^{\prime}, B_{i}^{\prime}$.

We now set

$$
\overline{\mathcal{G}}=\sum_{j \in \mathbb{Z}}\left(\overline{\mathcal{F}_{d_{j}}}+B_{j}+\widehat{u_{j}}+A_{j}^{\prime}\right) .
$$

In effect, $\overline{\mathcal{G}}$ is the sum

$$
\cdots+\overline{\mathcal{F}_{d_{0}}}+B_{0}+\widehat{u_{0}}+A_{0}^{\prime}+\overline{\mathcal{F}_{d_{1}}}+B_{1}+\widehat{u_{1}}+A_{1}^{\prime}+\overline{\mathcal{F}_{d_{2}}}+B_{2}+\widehat{u_{2}}+A_{2}^{\prime}+\cdots
$$

Clearly, the underlying order of each $\overline{\mathcal{F}_{d_{j}}}+B_{j}+\widehat{u_{j}}+A_{j}^{\prime}$ is isomorphic to an interval of $\mathbb{R}$ with a greatest point but no least point. So by proposition $3.3(2)$, $\overline{\mathcal{G}}$ can be assumed to have domain $\mathbb{R}$.

Let us check the requirements of the lemma. Requirement G1 is proved by induction as before. For G2, suppose that $x \in \widehat{u_{j}}$ for some $j \in \mathbb{Z}$. Referring to equation (6), $x$ lies just after a submodel $B_{j}$ of $\overline{\mathcal{G}}$ that is isomorphic to a final submodel of $\overline{\mathcal{C}_{j}}$. Take $y \in B_{j}$, so that $y<x$. Trivially, if $y<z<x$ and $\overline{\mathcal{G}}, z \models v$ then $v \in C_{j}$. Conversely, by G3 for $\overline{\mathcal{C}_{j}}$, for any $v \in C_{j}$ and $y<x$ there is $z \in B_{j}$ with $y<z<x$ and $\overline{\mathcal{G}}, z \models v$. Similarly, a copy of an initial submodel $A_{j}^{\prime}$ of $\overline{\mathcal{D}_{j}}$ can be found just after $x$, so all and only the elements of $D_{j}$ 'occur' arbitrarily near to $x$ on its right. Combining these two observations, we see that for any $v \in W$ we have $\overline{\mathcal{G}}, x \models\langle\partial\rangle v$ iff $v \in C_{j} \cup D_{j}=R\left(u_{j}\right)=R\left(f_{\mathcal{G}}(x)\right)$. Condition G2 for $x$ follows. Every other element $x \in \overline{\mathcal{G}}$ lies in an open interval of a structure $\overline{\mathcal{C}_{j}}$ or $\overline{\mathcal{D}_{j}}$ (of the form $\overline{\mathcal{F}_{d_{j}}}+B_{j}$ or $A_{j}^{\prime}+\overline{\mathcal{F}_{d_{j+1}}}$, respectively), so G2 holds inductively for $x$.

For G3, let $v \in \mathcal{G}$ and $x \in \mathbb{R}$ be given. Suppose that $x$ lies in the submodel $\overline{\mathcal{F}_{d_{i}}}+B_{i}+\widehat{u}_{i}+A_{i}^{\prime}$, say, of $\overline{\mathcal{G}}$. By assumption on the $u_{i}$, there are $j, k \in \mathbb{Z}$ with $j<i<k$ and $v \in W_{u_{j}} \cap W_{u_{k}}$. Let $y^{\prime}$ be the element of $\overline{\mathcal{G}}$ in the submodel $\widehat{u_{j}}$ (see equation (6)). So $y^{\prime}<x$. If $v=u_{j}$, then plainly $\overline{\mathcal{G}}, y^{\prime}=v$. If $v \neq u_{j}$ then $v \in R\left(u_{j}\right)$, so by G2 we have $\overline{\mathcal{G}}, y^{\prime} \models\langle\partial\rangle v$. Either way it is clear that $\overline{\mathcal{G}}, y \models v$ for some $y<x$. The case $z>x$ is similar, using $k$.

For $\mathrm{G} 4$, note that any leaf $v \in \mathcal{G}$ is equal to some $d_{j}$, and as equation (6) plainly shows, $\overline{\mathcal{F}_{d_{j}}}$ occurs as an interval in $\overline{\mathcal{G}}$. 
Now $\mathcal{F}$ is itself a connected generated subframe of $\mathcal{F}$, so by the lemma, $\overline{\mathcal{F}}$ can be found, with underlying order $\mathbb{R}$. Let $f=f_{\mathcal{F}}$. By property G3 of lemma 6.1 , $f: \mathbb{R} \rightarrow W$ is surjective.

Let $g: P V \rightarrow \wp(W)$ be an assignment into $\mathcal{F}$, and let $\mathcal{M}$ be the Kripke model $(W, R, g)$. Define $h: P V \rightarrow \wp(\mathbb{R})$ by $h(p)=f^{-1}(g(p))=\{x \in \mathbb{R}:$ $\mathcal{M}, f(x) \models p\}$. This gives us a linear model $M=(\mathbb{R},<, h)$.

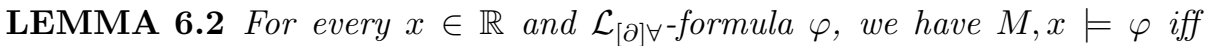
$\mathcal{M}, f(x) \models \varphi$.

Proof. By induction on $\varphi$. The atomic and boolean cases are easy. Assume the result for $\varphi$. Then $M, x \models \forall \varphi$ iff $M, y \models \varphi$ for every $y \in \mathbb{R}$, iff $\mathcal{M}, w \models \varphi$ for every $w \in W$ (inductively, and since $f$ is surjective), iff $\mathcal{M}, f(x) \models \forall \varphi$. Finally, $M, x=\langle\partial\rangle \varphi$ iff for every open interval $O \subseteq \mathbb{R}$ containing $x$, there is $y \in O \backslash\{x\}$ with $M, y \models \varphi$. Inductively, this holds iff for every open $O$ containing $x$, there is $y \in O \backslash\{x\}$ with $\mathcal{M}, f(y)=\varphi$. As $\mathcal{M}$ is finite, there are only finitely many values of $f$, so this is equivalent to saying that for some $w \in W$ with $\mathcal{M}, w \models \varphi$, every open $O$ containing $x$ contains a point $y \neq x$ with $f(y)=w$. This is plainly equivalent to $\overline{\mathcal{F}}, x \models\langle\partial\rangle w$ for some $w \in W$ with $\mathcal{M}, w \models \varphi$. By G2 of lemma 6.1 , this holds iff $R(f(x), w)$ for some $w \in W$ with $\mathcal{M}, w \models \varphi$ - that is, iff $\mathcal{M}, f(x) \models\langle\partial\rangle \varphi$.

THEOREM 6.3 (Lucero-Bryan, 2011) The $\mathcal{L}_{[\partial] \forall-\text { logic }} \mathrm{L}_{[\partial] \forall}$ of $\mathbb{R}$ is $\mathrm{KD}_{4} \mathrm{G}_{2} \cdot \mathrm{UC}$.

Proof. Again we leave it to the reader to check that $\mathrm{KD}_{4} \mathrm{G}_{2} . \mathrm{UC} \subseteq \mathrm{L}_{[\partial] \forall}$ (soundness). (Axiom $\mathrm{G}_{2}$ is valid over $\mathbb{R}$ because if $O$ is an open interval of $\mathbb{R}$ and $x \in O$, then $O \backslash\{x\}$ is not the union of three pairwise disjoint nonempty open sets [19, lemma 31].) For the converse (completeness), again we take an $\mathcal{L}_{[\partial] \forall \text {-formula }}$ $\varphi \notin \mathrm{KD}_{4} \mathrm{G}_{2} . \mathrm{UC}$ and show that $\neg \varphi$ is satisfiable over $\mathbb{R}$.

By $[12$, corollary 5.22$], \mathrm{KD}_{4} \mathrm{G}_{2} . \mathrm{UC}$ has the finite model property. (This is nontrivial and is proved by an unorthodox filtration in a style due to Shehtman [19]; see also [23].) So we may take a finite $\operatorname{Kripke}$ model $\mathcal{M}=(W, R, g)$ in which $\neg \varphi$ is satisfied, and such that the axioms of $\mathrm{KD} 4 \mathrm{G}_{2} . \mathrm{UC}$ are valid in the frame $(W, R)$. As usual, we may assume that $(W, R)$ is the frame $\mathcal{F}$ studied above. Let $M$ and $f$ be as above. As $f$ is surjective, we can take $x \in \mathbb{R}$ with $\mathcal{M}, f(x) \models \neg \varphi$. By lemma $6.2, M, x \models \neg \varphi$, and so $\neg \varphi$ is satisfiable over $\mathbb{R}$ as required.

We leave it as an exercise to show that $\mathrm{KD}_{4} \mathrm{G}_{2}$ is the logic of $\mathbb{R}$ in the

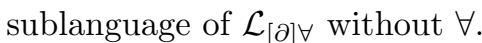

Once again, the proof transforms a finite Kripke model of a formula effectively into a model over $\mathbb{R}$ satisfying the formula, in a way that can be applied in practical examples.

EXAMPLE 6.4 The formula $\langle\partial\rangle p \wedge\langle\partial\rangle q \wedge \exists(\langle\partial\rangle q \wedge\langle\partial\rangle s \wedge[\partial] \neg p)$ is true at 0 in the Kripke model $\mathcal{M}$ shown in figure 1 . Its set of worlds is $\{0, \ldots, 4\}$, and the relation $R$ is indicated by the arrows; 0,1 are $R$-irreflexive and 2,3,4 are $R$-reflexive. Plainly, $\mathcal{M}$ is connected and locally 2 -connected. We select a sequence $\ldots, d_{0}, u_{0}, d_{1}, u_{1}, \ldots$ of elements of $\mathcal{M}$ as follows:

$$
\begin{array}{lccccccccccccccc}
\ldots & & u_{0} & & u_{1} & & u_{2} & & u_{3} & & u_{4} & & u_{5} & & u_{6} & \ldots \\
\ldots & d_{0} & & d_{1} & & d_{2} & & d_{3} & & d_{4} & & d_{5} & & d_{6} & & \ldots \\
\ldots & 2 & 0 & 3 & 1 & 4 & 1 & 3 & 0 & 2 & 0 & 3 & 1 & 4 & 1 & \ldots
\end{array}
$$




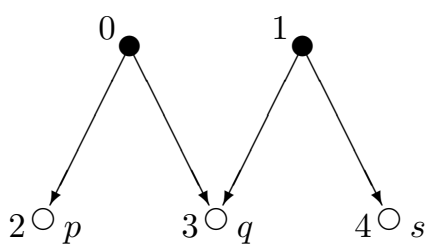

Figure 1: The model $\mathcal{M}$

This 'loops' over all points in $\mathcal{M}$ and meets the conditions in the proof above. In the notation of the proof, we have $C_{0}=\{2\}, D_{0}=C_{1}=\{3\}, D_{1}=C_{2}=\{4\}$, $D_{2}=C_{3}=\{3\}$, and so on. So $D_{i-1}=C_{i}=\left\{d_{i}\right\}$ for every $i \in \mathbb{Z}$.

We assume as usual that $\{0, \ldots, 4\} \subseteq P V$. Let $\mathcal{F}$ be the frame of $\mathcal{M}$, and define linear models

$$
\begin{aligned}
& P=\operatorname{Shuffle}(\{\widehat{2}\} ; \widehat{2}) \cong \overline{\mathcal{F}_{2}} \\
& Q=\operatorname{Shuffle}(\{\widehat{3}\} ; \widehat{3}) \cong \overline{\mathcal{F}_{3}} \\
& S=\operatorname{Shuffle}(\{\widehat{4}\} ; \widehat{4}) \cong \overline{\mathcal{F}_{4}}
\end{aligned}
$$

As $P$ is a shuffle, we can find a copy of it in the middle of itself, so $P \cong$ $P_{A}+P+P_{B}$ for some suitable $P_{A}$ and $P_{B}$, and similarly for $Q, S$. For each $i$, as $D_{i-1}=C_{i}=\left\{d_{i}\right\}$ we have $\mathcal{D}_{i-1}=\mathcal{C}_{i}=\mathcal{F}_{d_{i}}$, so

$$
\begin{aligned}
& \overline{\mathcal{C}_{0}}=\overline{\mathcal{F}_{d_{0}}}=\overline{\mathcal{F}_{2}}=P \cong P_{A}+P+P_{B} \\
& \overline{\mathcal{D}_{0}}=\overline{\mathcal{C}_{1}}=\overline{\mathcal{F}_{d_{1}}}=\overline{\mathcal{F}_{3}}=Q \cong Q_{A}+Q+Q_{B} \\
& \overline{\mathcal{D}_{1}}=\overline{\mathcal{C}_{2}}=\overline{\mathcal{F}_{d_{2}}}=\overline{\mathcal{F}_{4}}=S \cong S_{A}+S+S_{B} \\
& \overline{\mathcal{D}_{2}}=\overline{\mathcal{C}_{3}}=\overline{\mathcal{F}_{d_{3}}}=\overline{\mathcal{F}_{3}}=Q \cong Q_{A} \cong Q+Q_{B} \\
& \overline{\mathcal{D}_{3}}=\overline{\overline{\mathcal{C}_{4}}}=\overline{\mathcal{F}_{d_{4}}}=\overline{\mathcal{F}_{2}}=P \cong P_{A}+P+P_{B} \\
& \overline{\mathcal{D}_{4}}=\overline{\mathcal{F}_{d_{5}}}=\overline{\mathcal{F}_{3}}=Q \cong Q_{A} \cong Q+Q_{B}
\end{aligned}
$$

and so on. Equation (5) in the proof of lemma 6.1 tells us to write $\overline{\mathcal{C}_{i}} \cong$ $A_{i}+\overline{\mathcal{F}_{d_{i}}}+B_{i}$ and $\overline{\mathcal{D}_{i}} \cong A_{i}^{\prime}+\overline{\mathcal{F}_{d_{i+1}}}+B_{i}^{\prime}$, for each $i$ and for suitable $A_{i}, B_{i}, A_{i}^{\prime}$, $B_{i}^{\prime}$. So we can take

$$
\begin{aligned}
& \ldots B_{0}=P_{B} \quad A_{0}^{\prime}=Q_{A} \\
& B_{1}=Q_{B} \quad A_{1}^{\prime}=S_{A} \\
& B_{2}=S_{B} \quad A_{2}^{\prime}=Q_{A} \\
& B_{3}=Q_{B} \quad A_{3}^{\prime}=P_{A} \\
& B_{4}=P_{B} \quad A_{4}^{\prime}=Q_{A} \quad \ldots
\end{aligned}
$$

According to equation (6) in the proof, we define the linear model

$$
\begin{aligned}
& \overline{\mathcal{F}}=\sum_{j \in \mathbb{Z}}\left(\overline{\mathcal{F}_{d_{j}}}+B_{j}+\widehat{u_{j}}+A_{j}^{\prime}\right) \underbrace{}_{j=0} \\
&=\cdots+\underbrace{P+P_{B}+\widehat{0}+Q_{A}}_{j=1}+\underbrace{Q+Q_{B}+\widehat{1}+S_{A}}_{j=3}+\underbrace{S+S_{B}+\widehat{1}+Q_{A}}_{j=2} \\
&+\underbrace{Q+Q_{B}+\widehat{0}+P_{A}}_{j=4}+\underbrace{P+P_{B}+\widehat{0}+Q_{A}}_{j=3}+\cdots
\end{aligned}
$$


In this example, the expression obviously simplifies to

$$
\overline{\mathcal{F}} \cong \cdots+P+\widehat{0}+Q+\widehat{1}+S+\widehat{1}+Q+\widehat{0}+P+\widehat{0}+Q+\cdots
$$

and if we assign $p$ to the set of points in copies of $P$, and similarly for $q, s$, we obtain an entirely sensible and reasonable linear model $M$ over $\mathbb{R}$ in which $\langle\partial\rangle p \wedge\langle\partial\rangle q \wedge \exists(\langle\partial\rangle q \wedge\langle\partial\rangle s \wedge[\partial] \neg p)$ is true at any point in a copy of $\widehat{0}$.

\section{Conclusion}

We have proved completeness theorems for some 'spatial' logics over $\mathbb{R}$ in a fairly simple way. Spatial logic is of burgeoning interest and the methods used here may find further application. For example, there is potential for model checking a formula against a description of a model over $\mathbb{R}$ using shuffles and other operators, and this has already been explored for temporal logic in [6]. Some of the theorems that we have reproved here were originally proved in more general forms, for certain topological spaces. It remains to be seen whether the methods of this paper can be adapted to apply in this generality.

\section{Acknowledgments}

The author thanks the organisers for inviting him to the conference, Rob Goldblatt for handling the paper, Valentin Shehtman for helpful bibliographic information, and the referee for finding several mistakes in the submitted paper and for very detailed comments and suggestions.

\section{References}

[1] M. Aiello, J. van Benthem, and G. Bezhanishvili, Reasoning about space: the modal way, J. Logic Computat. 13 (2003), 889-920.

[2] G. Bezhanishvili and M. Gehrke, A new proof of completeness of S4 with respect to the real line, Tech. Report PP-2002-06, ILLC, Amsterdam, 2002.

[3] J P Burgess and Y Gurevich, The decision problem for linear temporal logic, Notre Dame J. Formal Logic 26 (1985), no. 2, 115-128.

[4] A Chagrov and M Zakharyaschev, Modal logic, Oxford Logic Guides, vol. 35, Clarendon Press, Oxford, 1997.

[5] K Doets, Monadic $\pi_{1}^{1}$-theories of $\pi_{1}^{1}$-properties, Notre Dame Journal of Formal Logic 30 (1989), 224-240.

[6] T. French, J. McCabe-Dansted, and M. Reynolds, Synthesis and model checking for continuous time: Long version, Tech. report, CSSE, University of Western Australia, 2012, http://www.csse.uwa.edu.au/ mark/ research/Online/sctm.htm.

[7] Y Gurevich, Monadic second-order theories, Model-Theoretic Logics (J Barwise and S Feferman, eds.), Springer-Verlag, New York, 1985, pp. 479-507. 
[8] P. Kremer, Strong completeness of $S_{4}$ wrt the real line, manuscript; http://individual utoronto.ca/philipkremer/onlinepapers/ strongcompletenessR.pdf, 2012.

[9] A. Kudinov, Topological modal logic of $\mathbb{R}$ with inequality, Russian Mathematical Surveys 63 (2008), 163-166.

[10] Tamar Lando and Darko Sarenac, Fractal completeness techniques in topological modal logic: Koch curve, limit tree, and the real line, preprint, http://philosophy.berkeley.edu/file/698/ FractalCompletenessTechniques.pdf, 2011.

[11] H Läuchli and J Leonard, On the elementary theory of linear order, Fundamenta Mathematicae 59 (1966), 109-116.

[12] J G Lucero-Bryan, The d-logic of the real line, J. Logic Computat. (2011), online, doi:10.1093/logcom/exr054.

[13] J.C.C. McKinsey and A. Tarski, The algebra of topology, Annals of Mathematics 45 (1944), 141-191.

[14] Grigori Mints, A completeness proof for propositional $S_{4}$ in Cantor space, Logic at Work (E. Orłowska, ed.), Studies in Fuzziness and Soft Computing, vol. 24, Physica-Verlag, Heidelberg/New York, 1998, Essays dedicated to the memory of Elena Rasiowa. ISBN 3-7908-1164-5, pp. 79-88.

[15] Grigori Mints and Ting Zhang, A proof of topological completeness for $\mathrm{S}_{4}$ in (0,1), Ann. Pure. Appl. Logic 133 (2005), 231-245.

[16] J. M. Plotkin (ed.), Hausdorff on ordered sets, History of Mathematics, vol. 25, American Mathematical Society, Providence, R.I., 2005.

[17] Alexander Rabinovich, Composition theorem for generalized sum, Fundam. Inf. 79 (2007), no. 1-2, 137-167.

[18] J G Rosenstein, Linear orderings, Academic Press, New York, 1982.

[19] V B Shehtman, Derived sets in euclidean spaces and modal logic, Tech. Report X-1990-05, University of Amsterdam, 1990.

[20] _ 'Everywhere' and 'here', Journal of Applied Non-classical Logics 9 (1999), 369-379.

[21] Modal logic of topological spaces, habilitation thesis, Moscow, 2000, In Russian.

[22] S Shelah, The monadic theory of order, Annals of Mathematics 102 (1975), 379-419.

[23] M. Zakharyaschev, A sufficient condition for the finite model property of modal logics above K4, Logic J. IGPL 1 (1993), 13-21. 